\title{
LA ENSENAANZA DE LA HISTORIA: RETOS Y DESAFÍOS ACTUALES
}

\author{
Carlos Gregorio López Bernal \\ Docente - Investigador
}

UES

Creo que la presentación que hizo el señor Ministro de Educación nos deja claro el camino o el proceso que hemos venido recorriendo desde hace un tiempo, en el marco del proceso de formación y actualización docente, cuya apuesta es fortalecer el dominio de los docentes en las áreas de las ciencias sociales que se hacen presentes en los programas de Estudios Sociales y Cívica.

Debe aclararse que ese programa de formación está bastante condicionado por los programas de estudio vigentes en educación básica y media. De hecho se partió básicamente de ellos. Lo que se busca entonces es actualizar, proporcionar conocimientos para el cuerpo docente, pero ya desde ahí nos enfrentamos a una serie de dificultades que obviamente inciden en lo que se ha estado haciendo.

En primer lugar, hay una evidente contraposición entre la cantidad de contenidos que se pretende desarrollar en esos programas de formación versus la profundización del conocimiento. Este es un problema siempre 
presente: lo vivimos en la universidad, lo vivimos en este proyecto del Plan de Formación Docente, lo viven los maestros día a día en las aulas. Adicionalmente, tenemos que decir que ha habido poco espacio para tratar con más detalle, con más cuidado, el tema de las metodologías de trabajo en el aula.Y es que, al final de cuentas, todos estos procesos se resuelven en el aula, cuando el maestro está con sus estudiantes en el escenario que sea: San Salvador como ciudad principal o cualquier pueblo o cantón de nuestro país.

Y ese trabajo en el aula está condicionado por varios factores, entre ellos: la formación inicial del docente en servicio, y en este punto, como bien señalaba el Coordinador del CENICSH, Lic. Carlos Enrique Rodríguez, al principio de este evento, el panorama es muy variable, pues nuestros docentes se forman en diversas universidades, incluso tenemos docentes que aún proceden de las Escuelas Normales. Hay que tomar en cuenta también el factor edad, hay docentes jóvenes y otros que están ya bastante mayores.

El segundo factor a considerar son los programas de estudio. A mi modo de ver están bastante atiborrados de contenidos y sin que exista un eje articulador bien definido que les dé sentido. Quienes hayan visto los programas de Estudios Sociales y Cívica a nivel de bachillerato saben a qué me refiero.

El tercer factor son los recursos didácticos. Aquí podemos pensar en libros de texto, libros de trabajo, bibliografia varia, acceso a internet, etcétera. Igual que en el caso de la formación docente el panorama también puede variar significativamente. Por último, pero el más importante quizás, la metodología de trabajo en el aula, que insisto se concreta a cada momento en cada aula de trabajo.

De la combinación de estos cuatro factores, tendremos al final, el resultado con los estudiantes sometidos a estos procesos. En la combinación de esos cuatro factores, ya el señor Ministro de Educación lo decía, el dominio que el docente tenga de su especialidad es clave. Los programas podrán condicionar, y de repente hasta se convierten en "camisa de fuerza", pero si el docente se mueve con seguridad en su ámbito de conocimiento, podrá 
hacer mucho.Y es en este proceso de profundización del conocimiento que hemos venido trabajando.

En ese marco quiero reflexionar sobre algunos de los retos que enfrenta la enseñanza de la historia.

La historia, como bien lo decía el Ministro de Educación, tiene un papel muy importante en la construcción de ciudadanía. Esto es algo que aparece recurrentemente en el trabajo que los historiadores hacemos. Lastimosamente, nuestro país tiene a la fecha un déficit muy alto en la construcción de ciudadanía. La historia nos ha ayudado para avanzar en la superación de este déficit, pero la historia también puede ayudar mucho para que los estudiantes desarrollen habilidades propias de la investigación, de la generación de conocimiento histórico.

La historia se presta mucho para la generación de competencias de análisis, de síntesis y de comunicación ya sea oral o escrita. ¿Por qué razón? Porque cuando nosotros estamos trabajando y hablando de historia en el aula, esos hechos del pasado no están desvinculados de la realidad del presente, pero la claridad de esa relación dependerá mucho del abordaje que se haga. ¿En qué sentido? que a través del tiempo diferentes sociedades, en contextos diferentes, enfrentan problemas similares. Si nosotros partimos de esta premisa, entonces cualquier problema histórico que estemos abordando inmediatamente nos remite al presente.

¿Qué tipo de problemas? Mencionemos uno básico: la producción; toda sociedad debe producir, ya sea para el consumo o para el intercambio. Otro problema que parecerá muy del presente pero que siempre ha estado se ha debido enfrentar es el uso de los recursos naturales, ¿con qué recursos naturales cuenta una sociedad para empeñarse en las actividades productivas?, ¿cómo los usa?, ¿quiénes se apropian de ellos?.Y si se produce, algo habrá que comerciar, intercambiar esos productos, aparece entonces otros problemas a resolver: carreteras, transportes, fronteras, etcétera. Si nos vamos a otro ámbito, resulta que cualquier sociedad medianamente compleja enfrenta el reto de gobernarse, de resolver conflictos, y según la época se resolverá de 
diferente manera, con mayor o menor participación, con mayor o menor concentración del poder. Una cosa que podrá parecer intrascendente: el problema de divertirse. En algún momento, los salvadoreños inventaron el mito de que eran muy trabajadores, al punto de que se vio el tiempo de ocio y divertimento, que son tan importantes para lograr una sociedad sana y con calidad de vida. Debiéramos conocer mejor cómo se divertían los salvadoreños de otras épocas, a lo mejor algo podríamos aprender para mejorar la vida de hoy.

Todos estos problemas se pueden estudiar desde la historia y desde las ciencias sociales: ¿qué es lo que cambia? Lo que cambia son las circunstancias, los recursos disponibles y los marcos de referencia para abordar y para analizar esos problemas. Pero la esencia de los problemas estará siempre presente.

Aquí tenemos una dificultad que enfrentan a diario los docentes en el aula: seguimos amarrados a trabajar por la vía de los contenidos y no por la vía de los problemas. Si nosotros planteamos un problema estamos retando al estudiante a enfrentarse a ellos. En cambio, si nosotros trabajamos por contenidos simplemente lo estamos obligando a hacer un estudio, a considerar ciertos elementos, pero no lo estamos retando.

Ya sea desde la especificidad de la historia o de la realidad social en general, plantear un problema obliga a proponer una forma de abordaje; dependiendo de la naturaleza del problema, así será la disciplina social más afin para ese abordaje. Si lo hacemos desde la historia, que es donde más yo me muevo, eso supone proponer o proporcionar al estudiante las fuentes documentales para estudiarlo y proponer una solución. Bajo esta lógica entonces resulta que un problema puede estar más vinculado a una esfera de la realidad, la política, por ejemplo. Pero ese mismo problema puede tener implicaciones económicas, históricas, sociales o hasta culturales. De ahí la necesidad de un enfoque integral para que el problema pueda estudiarse desde las competencias analíticas de las diferentes disciplinas sociales. 
En esta línea, entonces, creo que sí se puede hacer mucho desde la historia: contribuir a generar pensamiento crítico. La expresión es muy manida y de repente puede parecer hasta demasiado usada, pero nunca estará demás insistir en ella. Lo que nos falta muchas veces en la escuela, en la Universidad, en la sociedad misma, es justamente esa capacidad de pensamiento crítico.

Nos falta también mucha tolerancia, y desde las ciencias sociales, y la historia, se puede hacer mucho al respecto. Si pienso, por ejemplo, en la experiencia del siglo XIX, ¿qué resulta de esto? En el siglo XIX hubo un pensamiento hegemónico que nos obligó a pensar la sociedad en términos de que éramos una sociedad muy homogénea, al punto que se puso de moda un concepto muy interesante pero complicado: el mestizaje; somos una sociedad mestiza y dentro de la noción de mestizaje se diluyó todo: evadimos las diferencias y con ellas también las desigualdades y después pagamos caro esa omisión. En 1932, los indígenas se levantaron para recordarnos que estaban allí y que rechazaban la explotación y la marginación. La tolerancia implica la aceptación de la diversidad.

Desde la historia y las ciencias sociales se debe apuntar a trabajar la realidad social con rigurosidad y esta dependerá de dos cosas: el enfoque analítico y la evidencia con que estemos manejando ese enfoque analítico. Es decir, tampoco se vale en las ciencias sociales que se pueda decir cualquier cosa.

Hay otro reto muy importante que desde ya debiéramos tenerlo presente, y es que el devenir histórico de El Salvador se presta a la tentación de hacer un uso político del pasado y la historia es campo propicio para eso. Cuando digo uso político del pasado pienso en esa tentación de recurrir al pasado y a la historia para encontrar argumentos y evidencias que justifiquen o que apoyen una agenda de acción política del presente. La historia siempre está peleando con eso. Cualquier persona, grupo, partido político o gobierno, cae en la tentación de ver en el pasado aquello que soporte su visión del presente. Este es un riesgo inevitable. ¿Cuál puede ser el antídoto ante eso? Tenemos que enseñarles a nuestros jóvenes a pensar, a no tener visiones unilaterales o mecánicas de la realidad; es decir, no podemos pensar 
historias en blanco y negro. Solo si complejizamos la historia tendremos la posibilidad de lograr aprendizajes significativos y no simples compilaciones de sucesos y personajes.

Para ir concluyendo, creo que es importante tener claro que estamos frente a un proceso; cuando yo comencé a acercarme a esta iniciativa que tiene el Ministerio de Educación del Plan de Formación Docente, caí en la tentación de verlo de la manera tradicional: pensar que el problema está en el currículo, en los planes de estudio. Por suerte, el Ministro de Educación ha tenido una visión diferente y se ha optado por la formación y actualización como primer paso, dejando la reforma del currículo paro otro momento. A lo mejor por esta otra vía los resultados no serán tan inmediatos y tampoco generarán créditos políticos inmediatos, pero seguramente la calidad del trabajo en el aula sí cambiará, es más estoy seguro de que ya está cambiando. Si los docentes están mejor formados, más motivados y más comprometidos, podrán hacer mucho más con los mismos programas y los pocos recursos disponibles.

Para mi estos son los retos y sobre esto a lo mejor podamos discutir más adelante. Muchas gracias por su atención. 